\title{
UTILIZAÇÃO DE PAPAÍNA NO TRATAMENTO DE FERIDA E A VISITA DOMICILIAR COMO UMA FERRAMENTA PARA SISTEMATIZAÇÃO DA ASSISTÊNCIA DE ENFERMAGEM: Relato de Experiência
}

\author{
Simone Oliveira Ferreira \\ Universidade Estadual de Santa Cruz \\ simoneenfer@outlook.com \\ Nairan Morais Caldas \\ Universidade Estadual de Santa Cruz \\ nairanmorais@yahoo.com.br \\ Gisele D'Angela Camillo de Carvalho Rocha \\ Universidade Estadual de Santa Cruz \\ gisadangela@yahoo.com.br
}

\section{Resumo}

O presente estudo propõe relatar a experiência do uso da papaína durante o cuidado prestado a uma usuária do Sistema Único de Saúde (SUS) delineando toda assistência prestada a nível hospitalar e posteriormente nas visitas domiciliar que foi realizada no intuito de acompanhar e monitorar a evolução do processo de cicatrização da lesão, ademais com o objetivo de se promover o cuidado em uma perspectiva global. Este manuscrito contempla também a importância da assistência de enfermagem de forma sistematizada para um bom prognostico. Falar da visita domiciliar. Trata-se de um relato de experiência desenvolvido, a partir de uma ação extensionista da Universidade Estadual de Santa Cruz, vinculada ao Programa de Educação Superior pelo Trabalho para a Saúde, o qual foi cenário da vivência de uma acadêmica que atuou como bolsista. A análise dos dados produzidos ocorreu por meio da pesquisa nas bases de dados Lilacs e Scielo. Participou da experiência uma usuária diabética juntamente com a família, uma docente enfermeira e uma acadêmica do curso de Enfermagem. O cuidado em âmbito hospitalar ocorreu durante um mês e posteriormente a avalição do quadro clinico a nível domiciliar no período mensal que durou aproximadamente dois meses, onde se utilizou durante as trocas de curativo a papaína a 6\%, que corroborou para cicatrização da lesão, durante esse processo de cuidado foi estabelecido vínculo com a família acompanhada e o despertar da paciente para o autocuidado frente doença enfrentada. Finalizamos este relato salientando a importância do cuidado integral ao cliente durante a assistência hospitalar, bem como, do acompanhamento posterior no âmbito domiciliar, considerando a visita domiciliar como uma ferramenta para ampliação da assistência, aplicando terapêuticas eficazes e de baixo custo, a exemplo da papaína. Palavras-chave: Diabetes Mellitus. Visita domiciliar. Utilidade da papaína em feridas.

\section{USE OF PAPAINE IN WOUND TREATMENT AND THE HOME VISIT AS A TOOL FOR SYSTEMATIZATION OF NURSING ASSISTANCE: experience report}

\begin{abstract}
The present study proposes to report the experience of the use of papain during the care provided to a user of the Unified Health System (SUS), outlining all the care provided at the hospital level and later at the home visits that was performed in order to follow and monitor the evolution of the process of wound healing, in addition with the objective of promoting care in a global perspective. This manuscript also includes the
\end{abstract}


importance of nursing care in a systematic way for a good prognostic. Talk about the home visit. It is an experience report developed, based on an extension action of the Santa Cruz State University, linked to the Higher Education Program for Work for Health, which was the scene of the experience of an academic who acted as a scholarship holder. The analysis of the data produced occurred through the research in the databases Lilacs and Scielo. She participated in the experience with a diabetic patient along with her family, a nurse teacher and a nursing student. Hospital care occurred for one month and then the evaluation of the clinical picture at the household level in the monthly period, which lasted approximately two months, during which papain was used during dressing changes at $6 \%$, which corroborated for wound healing during this process of care was established bonding with the family accompanied and the awakening of the patient to self care before facing illness. We conclude by highlighting the importance of comprehensive care for the client during hospital care, as well as the subsequent follow-up at home, considering the home visit as a tool to increase care by applying effective and low-cost therapies, such as papain.

Keywords: Diabetes Mellitus. Home visit. Usefulness of papain in wounds.

\section{UTILIZACIÓN DE PAPAÍNA EN EL TRATAMIENTO DE FERIDA Y LA VISITA DOMICILIAR COMO UNA HERRAMIENTA PARA SISTEMATIZACIÓN DE LA ASISTENCIA DE ENFERMATE: Relato de Experiencia}

\section{Resumen}

A continuación le presentamos una relación con una experiencia de uso durante un período de cuidado y un uso único del Sistema Único de Salud (SUS) delineando toda la asistencia a un nivel hospitalario y una visita a su domicilio domiciliario que no sea un intuito de acompañar y monitorear y evolucionar. Procesamiento de la cicatrización de la información, junto con el objetivo de promover el cuidado en una perspectiva global. Este manuscrito contempla también la importancia de la asistencia de enfermería de forma sistematizada para el pronóstico. Falar da visita domiciliar. Trata de un relato de la experiencia de desarrollo, a partir de un curso de extensión de la Universidad Estadual de Santa Cruz, de la Oficina de Educación Superior del Trabajo para el Trabajo, o de la cenaria de la vida de la acadía de la acadía en el espacio. A análise dos dados produzidos se ha producido un error en las bases de datos de Lilacs and Scielo. Participa en la experiencia de la diabetes diabética con una familia, docente enfermaira y académica del curso de Enfermería. O cuidado en el hospital hospitalario ocorreu durante mi vida y luego en un quadro clínico a nivel nacional no durante los próximos meses, sino también como trocas de curativo a papaína a $6 \%$, corroborou para la cicatrización de la vida, detallada Es un proceso de cuidado para el cuidado de los animales con una familia acompañada y despertada del paciente para el autocuidado frente a la enfrentada. Finalizaremos esta relación con la importancia del cuidado integral del cliente durante la asistencia hospitalaria, el tratamiento, la orientación posterior, el tratamiento domiciliario, la visita domiciliaria y la ferramenta para la ampliación de la asistencia, la aplicación terapéutica, la administración de la información, el uso y el mantenimiento de la información.

Palavras-chave: Diabetes Mellitus. Visita domiciliaria. Utilidade da papaína em feridas.

Cidadania em Ação: Revista de Extensão e Cultura, Florianópolis (SC), v. 3, n.1, jan./jun. 2019. 


\section{INTRODUÇÃO}

A papaína é considerada como uma terapêutica efetiva no tratamento de feridas e seus benefícios são comprovados a partir das evidencias científicas registradas na literatura. Trata-se de uma mistura complexa de enzimas proteolíticas e peroxidasses presente no látex do vegetal carica papaya (mamão papaia) (SOUZA et al., 2017).

Assim, deparamo-nos em campo de prática com a papaína que se destaca pelo seu baixo custo final, apresenta pouco ou nenhum efeito colateral, facilidade de aplicação, além de uma ação similar à de debridantes químicos autos líticos, contudo, com maior seletividade e eficácia na lise especifica dos tecidos mortos.

A papaína possui ação diferenciada no desbridamento químico provocando, em doses diminutas, a proteólise, ou seja, a dissociação de uma quantidade importante de proteínas em moléculas mais simples e, por fim, em aminoácidos (SOUZA et al., 2017).

A indicação de seu uso é baseada em várias concentrações no decorrer das características de cada fase em que se encontra a lesão. Em casos de feridas secas ou com tecido de granulação a indicação na literatura é de que as concentrações de papaína devem variar de $2 \%$ a e $4 \%$, quando da presença de exsudato purulento, e quando da presença de tecido necrótico abundante recomenda-se a utilização de papaína na concentração de 10\% (LEITE et al. 2012).

No que se refere à atenção domiciliar a pessoas com ferida ocasionada pela diabetes, gera na promoção de atenção integral aos fatores que corroboram na recuperação do quadro clinico e na prevenção de eventuais complicações que tal patologia pode acarretar (CAIAFA; 2011; CUNHA; GAMA, 2012).

Assim, a Visita domiciliar define-se como um conjunto de ações de saúde direcionadas para o atendimento tanto educativo como assistencial, possibilitando um processo interativo mais eficiente entre os membros da equipe de saúde com a família, conhecendo a realidade enfrentada pela mesma, contribuindo para a integralidade da assistência (SANTOS; MORAES, 2011).

Portanto, o presente estudo teve como objetivo relatar a experiência da visita domiciliar para o tratamento de uma ferida com o uso da papaína; retratar a importância da assistência de enfermagem de forma sistematizada para um bom prognostico da lesão. 


\section{METODOLOGIA}

Esta pesquisa constitui um relato de experiência, desenvolvido a partir de uma ação extensionista da Universidade Estadual de Santa Cruz, vinculada ao Programa de Educação Superior pelo Trabalho para a Saúde, que é um programa interministerial (Saúde e Educação) direcionado ao fortalecimento de áreas estratégicas para o Sistema Único de Saúde - SUS.

O programa tem como fundamento a educação pelo trabalho, considerada uma das estratégias do Programa Nacional de Reorientação da Formação Profissional em Saúde, o PRÓ-SAÚDE, em implementação no Brasil desde 2005.

O manuscrito faz parte de um subprojeto aprovado pelo Comitê de Ética da Universidade Estadual de Santa Cruz, protocolo no 365/10, cujo título da pesquisa é: "Processo de Construção das linhas de cuidado do município de Ilhéus/Bahia”. Descreve aspectos vivenciados pela acadêmica de enfermagem que atuou como bolsista, desenvolvendo a pesquisa rede de média complexidade que oferece ações e serviços, de saúde, aos usuários portadores de diabetes e hipertensão no município de Ilhéus.

O corpus do estudo foi composto por uma usuária acompanhada pelo Centro de Atendimento do Diabético, Hipertenso e Idoso, do município de Ilhéus (CADHII), com idade de 35 anos, portadora de diabetes. A usuária e residente no município de Ilheus, a aceitação em participar do estudo ocorreu mediante assinatura do Termo de Consentimento Livre e Esclarecido (TCLE) de acordo com a resolução $n^{\circ}$ 466/12.

Por meio da investigação da história natural da doença soube que a mesma iniciou com uma ferida que se apresentou como um pequeno ferimento na região abdominal, posteriormente devido ao quadro de hiperglicemia evoluiu para um abcesso, ocasionando deiscência. Foi abordada durante os cuidados prestados em âmbito hospitalar que durou um mês e após a alta os cuidados foram direcionados durante as visitas domiciliares que ocorreu no período de agosto a setembro de 2016.

Inicialmente, procurou-se explicar o objetivo do estudo, comentando que todo acompanhamento constituiria em uma oportunidade de aplicar os benefícios da papaína e corroborar para rápida cicatrização da lesão, com intuito de promover a recuperação da cliente, destaca-se que a mesma poderia desistir a qualquer momento, conforme sua vontade.

Assim a senhora recebeu os primeiros cuidados no âmbito hospitalar por conta da gravidade do quadro apresentado. Com a introdução da papaína durante a assistência, a mesma teve alta, sendo os cuidados direcionados para visita domiciliar, a família participou do processo de cuidado, onde a acadêmica de enfermagem juntamente com a enfermeira do (CADHII) realizava as trocas de 
curativos, orientando por meio de ações educativas sobre prevenção de futuras lesões.

A orientação foi direcionada para como deveria ocorrer as trocas de cobertura oclusiva, destacando a promoção da independência da cliente, para que a mesma aprendesse o autocuidado dentro do processo saúde-doença, também com o intuito de que os familiares aprendessem a fornecer o suporte necessário, para que a cliente evoluísse para um bom prognóstico. A supervisão e orientação se deu por meio de uma profissional enfermeira do (CADHII), juntamente com a bolsista do projeto.

Realizou-se observação contínua da participante e educação em saúde no decorrer dos encontros diários que duravam, em média, 40 minutos. Investigou-se também o prontuário da cliente para coleta de informações sobre o quadro clínico, além do registro fotográfico, a evolução de enfermagem diária durante a visita domiciliar e procedimentos realizados.

A busca na literatura para fundamentação teórica deste relato ocorreu nos meses de agosto a outubro de 2015, através das seguintes bases de dados: Scielo (Scientific Electronic Library Online), LILACS (Literatura Latino-Americana e do Caribe em Ciências da Saúde), utilizando-se dos descritores: Diabetes Mellitus, Visita domiciliar, Utilidade da papaína em feridas.

\section{RESULTADOS}

Usuária de 35 anos, diagnosticada com DM tipo 2 há 5 anos, casada, reside com o esposo e os filhos em seu domicílio, no município de Itabuna no interior da Bahia. Referiu durante a visita de enfermagem que inicialmente surgiu uma ferida de pequeno porte na região abdominal, realizou tratamento por meio de pomadas, contudo sem obter sucesso.

Após alguns dias observou que a ferida evoluía para um odor desagradável e buscou atendimento na Unidade de Saúde da Família (USF) do município em que reside. Após os profissionais da USF observarem que a ferida já estava evoluindo para um abscesso encaminhou a usuária para o hospital de referência da cidade para que fosse realizado um tratamento mais intensivo.

Como tratamento para lesão desse porte, a papaína surgiu como uma terapêutica a partir do olhar clínico da enfermeira estomaterapeuta que acompanhou a evolução do caso junto com a equipe médica e a discente bolsista, realizou-se também o controle glicêmico da paciente, o uso de antibióticos, alimentação equilibrada e terapêutica com antidiabéticos orais. 
O procedimento do curativo se estabeleceu inicialmente através da inspeção e análise da lesão. Posteriormente, classificou-se a lesão quanto as suas características para a seleção da cobertura a ser utilizada. Os curativos a base de papaína foram realizados uma vez ao dia, no início das atividades práticas do estágio, no período da manhã, e quando necessário. Aplicava-se o soro fisiológico a 0,9\% em jato para limpeza previa da ferida, após isso, realizou-se a cobertura com gaze estéril embebida na solução de papaína a 6\% sobre a lesão, visto que o leito da ferida apresentava exsudato purulento; fechamento do curativo com micropore para evitar a exposição da papaína ao ar e a luz (prevenindo queimaduras e neutralização da enzima).

A partir de então, ocorreu o monitoramento da evolução da ferida. Após duas semanas utilizando a terapêutica proposta no hospital, observou-se que o leito da ferida apresentava tecido de granulação, formado por tecido conjuntivo denso, e as bordas da ferida já estavam próximas.

A paciente apresentou alta hospitalar após um mês de cuidados intensivos, e assim, continuou-se o acompanhamento da mesma no âmbito domiciliar. Utilizando a mesma terapêutica aplicada em âmbito hospitalar e monitorando o nível glicêmico e pressórico, fazendo as orientações necessárias acerca do padrão dietético e do cuidado diário nas trocas de curativos.

Transcorridas um mês de cuidado no domicilio, foi constatado que a ferida apresentava tecido de granulação, com ausência de secreção purulenta. A partir desse ponto, ocorreu a limpeza da ferida com soro fisiológico e cobrindo-a com gaze estéril. Observou-se uma evolução por completo no estado geral da paciente, não apenas no que se refere ao prognóstico satisfatório da lesão que já estava fechando e cicatrizando, mas também no que se refere à qualidade de vida da usuária e do processo de empoderamento frente ao autocuidado e do entendimento da doença.

A visita domiciliar acolheu as diferentes necessidades apresentada pela cliente, contudo a experiência aqui relatada apresentou algumas limitações, pois não foi possível acompanhar a cicatrização total da ferida devido ao encerramento do período de prática. Entretanto, o pouco período de cuidado prestado à usuária e sua família pode promover cicatrização com boa epitelização em dois meses de acompanhamento, além de constatar os benefícios que a papaína promove na evolução de feridas diabéticas.

\section{CONCLUSÃO}

$\mathrm{O}$ vínculo promovido pela visita domiciliar promove uma assistência de enfermagem diferenciada, ou seja, mais holística, além de sensibilizar o indivíduo-família quanto à necessidade 
de cuidados necessários para uma vida com mais qualidade frente ao processo da doença.

Vale ressaltar, que essa pesquisa demonstrou que a cliente chegou a desenvolver uma lesão ocasionada pela falta de controle do quadro glicêmico, sabemos, pois que a diabetes é uma doença crônica grave que precisa de um acompanhamento que deve ser promovido pela Unidade Básica de Saúde (UBS), assim destaca-se aqui a falha do serviço em não acompanhar de forma continua a cliente, e um descuido da própria usuária em não procurar o primeiro nível de assistência para o monitoramento de sua saúde, assim percebe-se cada vez mais a importância que a atenção primária representa na saúde coletiva, uma vez que, se a cliente tivesse procurado a UBS para controlar a glicemia e receber todos os cuidados de forma integral teria evitado a ocorrência da ferida que levou a um processo de decência e o ônus que ocasionou a um transtorno emocional para usuária.

Finalizamos este relato salientando a importância do cuidado integral durante a assistência hospitalar, bem como, do acompanhamento da usuária e da ferida no âmbito domiciliar, aplicando terapêuticas eficazes e de baixo custo, a exemplo da papaína. 


\section{REFERÊNCIA}

BOEIRA, G. S. et al. Atuação interdisciplinar para pacientes com diabetes na atenção básica: uma revisão bibliográfica. Rev Epidemiol Control Infect, Santa Maria, RS, v. 2, n. 4, p.141143, 2012.

CAIAFA, J. S. et al. Atenção integral ao portador de pé diabético. Jornal Vascular Brasileiro, [s.1.], v. 10, n. 42, p.1-32, 2011.

JÚNIOR, F. et al. Programa hiperdia: do preconizado ao realizado - interfaces com a ética na enfermagem. Rev Enferm FACEMA, 2011. Disponível em:

http://189.59.9.179/cbcenf/sistemainscricoes/arquivosTrabalhos/. Acesso em: 10 de out. 2014.

LEITE, A.P.; et al. A efetividade de um protocolo de uso do gel de papaína a $2 \%$ e $4 \%$ na cicatrização de úlceras venosas. Niterói, 2012.

SANTOS, E.M.; MORAES, S. H. G. A visita domiciliar na estratégia saúde da família:

Percepção de enfermeiros. Cogitare Enferm, Curitiba, v. 16, n. 3, p. 492- 497, jul./set. 2011.

SOUZA, M.C.A.; et al. Úlcera crônica tratada com gel de papaína 10\% na Estratégia Saúde da Família: relato de experiência. Rev Bras Med Fam Comunidade, Rio de Janeiro,v. 12, n.39, p. 1-8, jan./dez., 2017.

TORRES, H.C. et al. Avaliação estratégica de educação em grupo e individual no programa educativo em diabetes Rev Saúde Pública, São Paulo, v. 43, n.2, p.291-298, 2009. Disponível em : http://www.scielo.br/scielo.php.Acesso em: 16 out. 2014. 